\title{
The impact of antimicrobials on gut bacteria: an interview with Karen Scott
}

\author{
Karen P Scott*,1 \\ ${ }^{1}$ Gut Health Group, The Rowett Institute, University of Aberdeen King's College, Aberdeen, AB24 3FX, UK \\ *Author for correspondence: k.scott@abdn.ac.uk
}

\author{
“Going forward, I think more targeted therapies will come into play instead of the \\ broad-spectrum antibiotics widely used now"
}

First draft submitted: 9 April 2019; Published online: 12 September 2019

In this exclusive interview, Karen Scott discusses how antimicrobials affect the gut microbiome and the consequences of this for patient clinical outcome. This interview was conducted by Ellen Colvin, Editor of Future Microbiology.

Karen Scott is a Senior Research Fellow at the Rowett Institute, University of Aberdeen. She leads a research team investigating the molecular mechanisms by which key members of the gut microbiota utilize specific dietary components including prebiotics and fiber. Another interest is the development of the gut microbiota throughout life, from birth and the impact of diet on this process. In vitro bacterial growth studies utilizing our large culture collection of gut anaerobes (in pure culture, mixed culture and fermenter systems) and bioinformatic analyses illustrate niche-specific processes and bacterial interactions. Resident bacteria also act as an important reservoir of transferable antimicrobial resistance genes, and other work investigates the flow of resistance from farm to fork.

\section{Your research is focused on the impact of antimicrobials on gut bacteria. What key findings provide evidence for this?}

Several studies now published have looked at the effect of antimicrobials on gut microbiota and the immediate effect they have. As most antimicrobials are not specific, they will decrease the numbers of the pathogenic bacteria for which they are given, however, they will also influence commensal gut microbiota. The published studies have demonstrated that immediately after antibiotic treatment, the composition of the gut microbiota is dramatically changed. What is most interesting is that when treatment is ceased, for example, with antibiotics, the gut microbiota composition recovers almost to where it was before, but importantly, not exactly. Thus, we are now becoming more aware that specific bacterial strains or species are more susceptible to antibiotic effects, and these can sometimes be significant bacteria. This means that while the general microbiota recovers, there may be specific functions that remain absent.

\section{How does this impact patient recovery?}

The most obvious example of this concerns Clostridium difficile infection. This is a direct consequence of antibiotics decimating the 'normal' microbiota and enabling this facultative pathogen to establish as a general member of the community and overcome everything else, becoming very abundant. This can then have serious consequences for patient health.

\section{How do antimicrobials impact resistance? Is this having a larger impact on patients than we realize?}

We know that when you apply a selective pressure to bacteria, they do their best to survive that selective pressure, often through the transfer of resistance genes. This is not unique to any single country, it is happening everywhere and multidrug-resistant pathogens are occuring with increasing frequency. Furthermore, resistance genes are not unique to either a specific mammalian host or a specific bacterial species, they can cross species barriers very easily. A caveat to this is seen whereby certain types of antibiotic resistance genes are more prevalent in specific bacterial types. For example, Gram-negative bacteria or Gram-positive bacteria each tend toward certain antibiotic mechanisms

Future Medicine 
because of their specific structures. Certain efflux pumps are more prevalent in Gram-negatives, but these bacteria are equally likely to be present in a cow or in a human.

\section{What are your predictions for the next 10-15 years in the field of gut microbiomes \& antimicrobials? What do you hope to see?}

As our understanding of the broad-spectrum effect of antibiotics increases, we are much more conscious of using them responsibly. Importantly, antibiotics are still required as they are vital for treating disease, but for us to keep this as an option, we must only use them when absolutely necessary and be careful to ensure we do not overuse them. Going forward, I think more targeted therapies will come into play instead of the broad-spectrum antibiotics widely used now. Many groups are working on utilizing commensal bacterial activities against pathogenic bacteria. For example, bacteriocins for $C$. difficile. This is where the focus is now, in trying to establish more targeted approaches and I believe this will continue in the future. This will allow us to reserve important existing antibiotics to eliminate specific pathogens, when there are no other options.

\section{Disclaimer}

The opinions expressed in this interview are those of Karen Scott and do not necessarily reflect the views of Future Medicine Ltd.

\section{Financial \& competing interests disclosure}

The author has no relevant affiliations or financial involvement with any organization or entity with a financial interest in or financial conflict with the subject matter or materials discussed in the manuscript. This includes employment, consultancies, honoraria, stock ownership or options, expert testimony, grants or patents received or pending, or royalties.

No writing assistance was utilized in the production of this manuscript. 